Case report

\title{
Two cases of primary leiomyosarcoma of sigmoid colon treated with laparoscopic surgery: A case report and a review of literature
}

\author{
Alimohammad Bananzadeh ${ }^{\mathrm{a}}$, Maral Mokhtari ${ }^{\mathrm{b}}$, Maryam Sohooli ${ }^{\mathrm{c}}$, Ramin Shekouhi ${ }^{\mathrm{c}, *}$ \\ ${ }^{\text {a }}$ Colorectal Research Center, Department of Surgery, Shiraz University of Medical Sciences, Shiraz, Iran \\ ${ }^{\mathrm{b}}$ Pathology Department, Shiraz University of Medical Sciences, Shiraz, Iran \\ ${ }^{c}$ Colorectal Research Center, Department of Surgery, Shiraz University of Medical Sciences, Shiraz, Iran
}

\section{A R T I C L E I N F O}

\section{Keywords:}

Colon cancer

Leiomyosarcoma

Sigmoid colon

Laparoscopy

\begin{abstract}
A B S T R A C T
Introduction and importance: Leiomyosarcoma (LMS) of the colon is an extremely rare and highly invasive tumor arising from the muscularis propria of the gastrointestinal tract. After the introduction of oncogenic role of KIT by immunohistochemistry (IHC), the reported cases of gastrointestinal leiomyosarcoma were highly limited. True LMS of the colon is such a rare disorder that there isn't much description of its nature.

Case presentation: We reported two very rare cases of primary leiomyosarcoma of sigmoid colon, which referred to our institution with symptoms of abdominal pain, lower GI bleeding and fatigue. After the initial investigations, both patients were diagnosed with primary LMS of sigmoid colon that underwent laparoscopic tumor resection.

Clinical discussion: The classical colonic LMS presents with a vast majority of non-specific symptoms including mild abdominal pain, fresh/obscure rectal bleeding, and weight loss. The most common location for colonic LMS is the sigmoid colon, and ascending colon. The prognostic factors for the disease outcome have not been established properly; however, patient age, tumor size/grade, and local/distant dissemination are of great importance.

Conclusion: Herein, we reported two rare cases of primary leiomyosarcoma of sigmoid colon that was treated with laparoscopic surgery.
\end{abstract}

\section{Background}

Leiomyosarcoma (LMS) of the colon is an extremely rare and highly invasive tumor arising from the muscularis propria of the gastrointestinal tract [1]. Before 1998, every neoplasm raised from mesenchymal cells was mistakenly classified as LMS; However, after the introduction of the oncogenic role of KIT by immunohistochemistry (IHC), only a very small number of true gastrointestinal leiomyosarcomas were reported [2]. Immunohistochemically, true LMS expresses actin (SMA) and desmin without the expression of GIST markers (CD117, CD34, and DOG1.1) and KIT mutations, which allow distinguishing LMS from other GI mesenchymal neoplasms $[1,3]$. We herein describe two rare cases of Leiomyosarcoma of sigmoid colon that were treated with laparoscopic resection. This study was reported in line with the SCARE criteria [4].

\section{Case presentation}

\subsection{Case 1}

We reported a case of a 48-year-old man referred to our surgical outpatient clinic in December 2019. He presented with mild intermittent abdominal pain and occasional rectal bleeding for eight months without any history of weight loss, fatigue or night sweating. Furthermore, He had no family history of malignant neoplasia. General examination and vital signs were normal. Abdominal examinations revealed an illdefined, round, fixed mass at the left lower quadrant. Digital rectal examination was normal. Routine lab data were unremarkable, except mild microcytic anemia that was due to his thalassemia trait (Mentzer index $=13.46$ ). Carcinoembryonic antigen (CEA) test was normal. After the initial investigation patient underwent colonoscopy that was suggestive of a large polypoid mass with hard texture in $15 \mathrm{~cm}$ from the anal

\footnotetext{
* Corresponding author at: Department of Surgery, Colorectal Research Center, Shiraz University of Medical Sciences, Zand Avenue, P.O. Box 71345-1744, Shiraz, Iran.

E-mail address: shekouhi.ramin@gmail.com (R. Shekouhi).
} 
verge; rest of the colon were unremarkable.

\subsubsection{Treatment}

Before the operation, patient was scheduled for a routine metastatic workup with Contrast-enhanced computed tomography (CT) of thoracic and abdomen and pelvis, and liver function test (LFT). The abdominal CT showed diffuse wall thickening of the sigmoid colon and distal part of the left colon associated with enhancing soft tissue mass measuring about $72 \mathrm{~mm} \times 40 \mathrm{~mm}$ in sigmoid colon that was suggestive of a malignant process (Fig. 1). Also, evidence of multiple regional lymph nodes was seen adjacent to the sigmoid colon. Chest CT and Liver function test (LFT) were unremarkable. The surgery was conducted under general anesthesia with the purpose of complete resection of the tumor and involved lymph nodes.

Laparoscopic anterior resection was performed with resection of sigmoid colon and upper part of rectum. Colorectal anastomosis was performed with no diverting ileostomy. Tumor dimensions were $8 \times 6 \times$ $4.5 \mathrm{~cm}$ with a smooth surface and the cross-section showed homogenous white appearance. The specimen was sent for IHC profiling and histologic evaluation (Fig. 2). Histopathologic examinations revealed a neoplasm consists of spindle cells with hyperchromatic nuclei and mild pleomorphism. Subsequent immunohistochemistry showed immunoreactivity for smooth muscle actin and desmin but the tumor cells were negative for S100, CD34, C-KIT and DOG-1. The Ki-67 index was $15-20 \%$. KIT mutation study also showed no mutation in exon 9, 11, 13 and 17. Also, histologic evaluations of resected lymph nodes showed reactive sinus histiocytosis with no evidence of tumoral involvement.

\subsubsection{Outcome and follow-up}

The post-operative course was uneventful without any signs of shortterm complications. The patient discharged four days after surgery. The patient was scheduled for Contrast-enhanced computed tomography (CT) of thoracic and abdomino-pelvic every 6 months, and colonoscopy every 12 months. Patient has no signs of local recurrence or distant metastasis Up-to-date. We will follow the patient for at least 5 years of close surveillance.

\subsection{Case 2}

On April 29th 2020, a 49-year-old man came with 3-month history of fatigue, melena, and unintentional weight loss. Family history was negative for any previous disease. General examination revealed
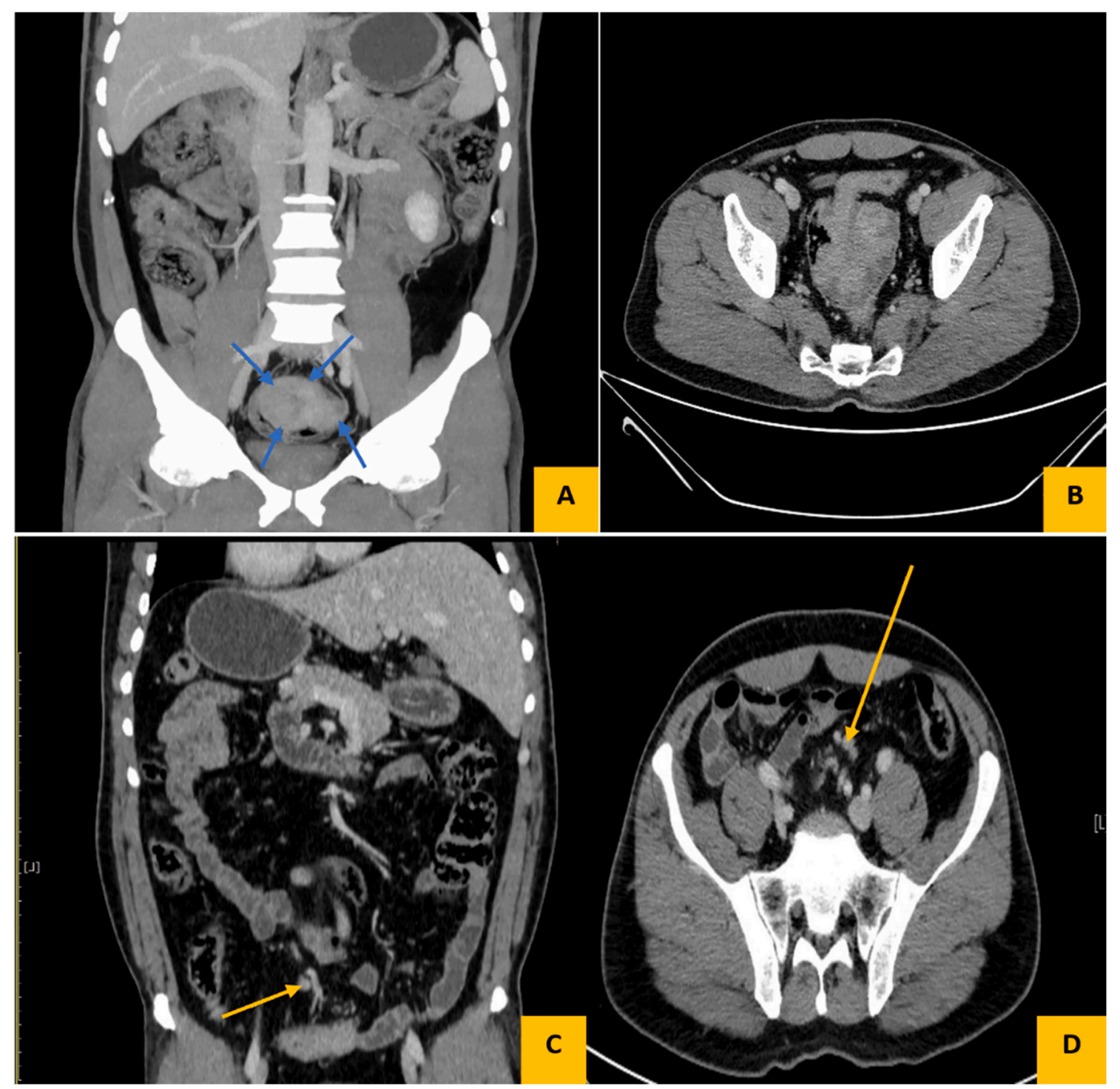

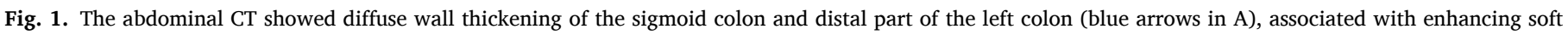

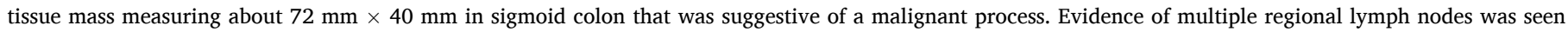
adjacent to the sigmoid colon (yellow arrows). 

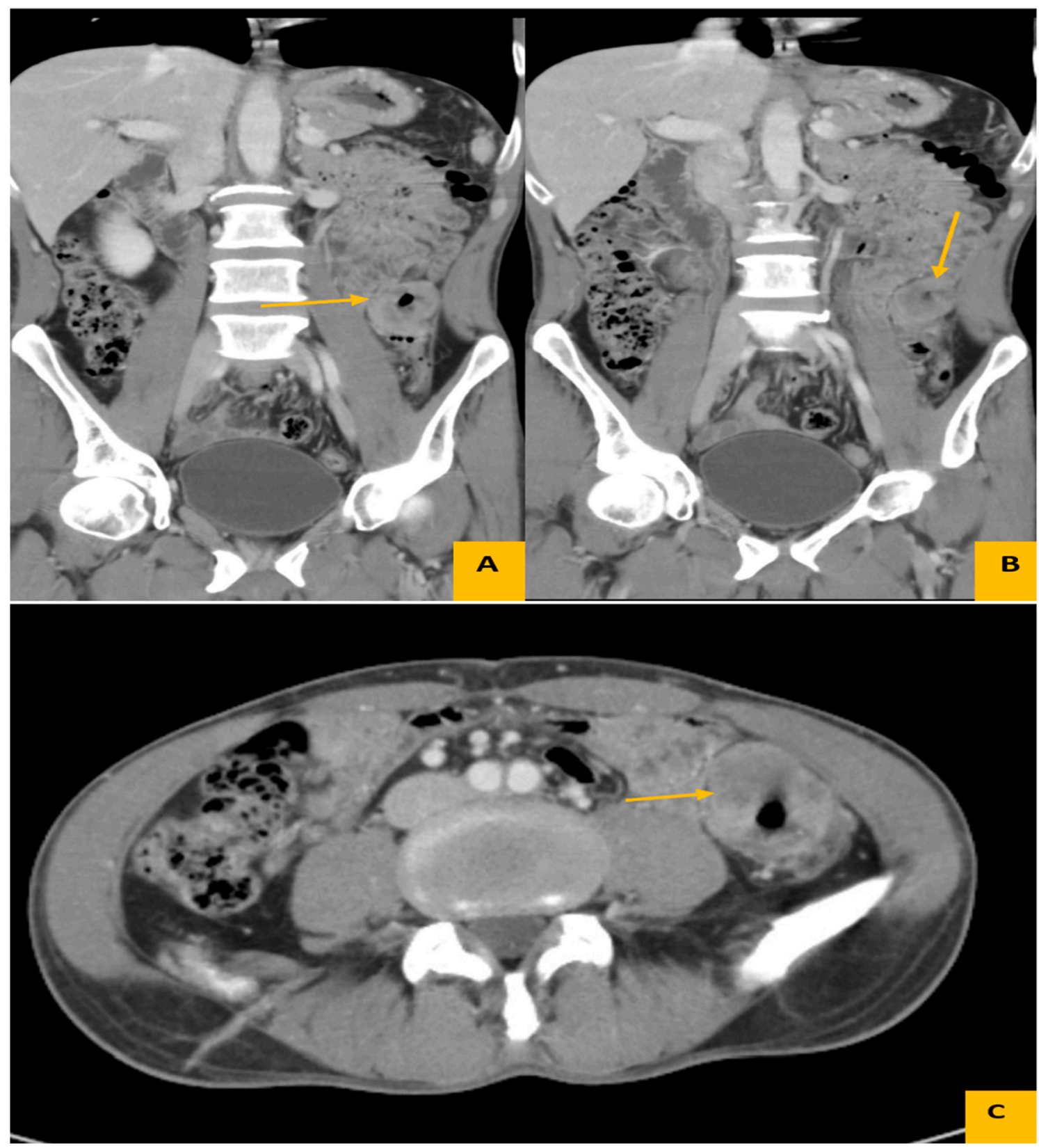

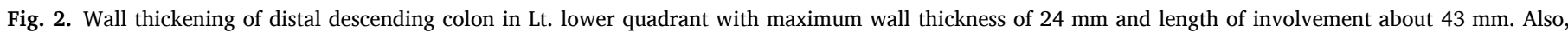
thickening of adjacent peritoneum was demonstrated.

temporal wasting and cachexia. Vital signs and abdominal examinations were unremarkable. Digital rectal examination showed grade-II internal hemorrhoids. Lab data was in favor of microcytic-hypochromic anemia $(\mathrm{HB}=11.7)$. Carcinoembryonic antigen (CEA) test was normal. Colonoscopy revealed a large $3 * 4 * 3.5 \mathrm{~cm}$ circumferential mass with deep central ulceration in sigmoid colon $40 \mathrm{~cm}$ from anal verge.

Prior to surgery, Abdomino-perineal CT showed circumferential wall thickening of distal descending colon in Lt. lower quadrant with maximum wall thickness of $24 \mathrm{~mm}$ and length of involvement about 43 $\mathrm{mm}$. Also, thickening of adjacent peritoneum without signs of lymph node metastasis were demonstrated (Fig. 2). Pre-op Chest CT was normal.

\subsubsection{Treatment}

Laparoscopic left hemicolectomy was conducted under general anesthesia with the purpose of complete resection of the tumor and involved lymph nodes. The specimen was extracted via a midline incision and extra-corporal anastomosis was performed. The specimen was sent for IHC profiling and histologic evaluation.

\subsubsection{Outcome and follow-up}

Surgery was successfully performed without any short-time complications and patient was discharged 4 days after the operation. Histologic evaluations were suggestive of spindle cells tumor with hyperchromatic nuclei and moderate pleomorphism (mitotic activity $>5 / 50 \mathrm{HPF}$ ). IHC results were positive for desmin and SMA. Ki-67 was positive in $15-20 \%$ of neoplastic cells. C-KIT, CD34, DOG1, and S100 were negative. Unfortunately, histologic evaluations obtained from surgery revealed peritoneal and abdominal wall involvement by high grade sarcoma. He was referred to an oncologist for chemotherapy initiation with Adriamycin, and Ifosfamide. Also, the patient was scheduled for Contrastenhanced computed tomography (CT) of Thoracic and Abdomino- 
pelvis every 6 months, and colonoscopy every 12 months. Follow-up CT scan revealed multiple small nodules in both lungs with the largest being 4-mm in lateral segment of right lower lobe in favor of metastatic lesions. Abdominal CT showed multiple malignant-looking lesion in peritoneal cavity with the largest being $38 * 39 \mathrm{~mm}$. Patient is alive and is under close follow up by an oncologist (Fig. 3).

\section{Discussion}

GISTs are the most common mesenchymal GI malignancies with the incidence of $1-3 \%$ of all GI malignancies. Moreover, LMS is an extremely rare cancer representing 3-6\% of all GI mesenchymal tumors. They arise from muscularis mucosae or propria occurring mostly in middle-aged males $[3,5]$. During the pre-KIT area, most of the GI mesenchymal malignancies were wrongfully diagnosed as leiomyoma, LMS, or leiomyoblastomas. However, their incidence declined after the diagnosis of KIT mutations and the immunohistochemical differences between LMS and other GI mesenchymal tumors, particularly GISTs [6].

In 1998, Hirota et al. first introduced the presence of activating KITmutations in $94 \%$ of GISTs [7]. Kit gene, a tyrosine kinase receptor proto-oncogene, causes increased cellular proliferation. Subsequently, this mutation can lead to cellular atypia and neoplasia. Later studies confirmed that 95\% of GISTs expressed CD34, CD137, and DOG1.1 [8]. On the contrary, the LMS is negative for kit-mutations and mostly positive for desmin, SMA, h-caldesmon, and vimentin [9].

Differentiation between LMS and GISTs is paramount importance since they have very similar clinical presentations but require radically different courses of treatment. The cellular origin of GISTs, the interstitial cells of Cajal, was first introduced in mid-1990s. However, LMS originates from muscle fibers of the muscularis mucosae and muscularis propria [10]. Also, most of the colonic LMS are polypoid, while esophageal LMS is mainly intramural [11].

The most common location of GI involvement by GISTS is the stomach (55\%), small intestine (29\%), colon (2.9\%), and rectum $(2.7 \%)$ [12]. However, GI LMS mainly involves stomach followed by small intestines, rarely colon and rectum [13]. True LMS of the colon is such a rare disorder that there isn't enough description of its nature. The classical colon LMS presents with a vast majority of non-specific symptoms including mild abdominal pain, fresh/obscure rectal bleeding, intraabdominal hemorrhage, weight loss, changes in bowel habits, bowel obstruction, and tenesmus [14]. Diagnosis is based on colonoscopy and histologic evaluation and IHC profiling.

Based on our survey of the four main online databases (EMBASE, PUBMED, MEDLINE, and Scholar), there are only thirty-four previous cases of published colonic LMS after the pre-kit era that was confirmed by immunohistochemistry (Table 1). Sigmoid colon, followed by the ascending colon, is most commonly involved by primary LMS. The prognostic factors for the disease outcome have not been established properly, however patient age, tumor size/grade, and local/distant dissemination are of great importance [15]. Yamamoto et al., concluded that the only negative predictive factor for survival is tumor size of more than $5 \mathrm{~cm}$ [16]. However, based on previous case studies local and distal recurrence occurred even with favorable tumor features [14]. Compared with adults, infantile LMS has a better prognosis even with poor histologic features [17].

According to the recent survey by Faraj et al. lymph node involvement is very unlikely and distant metastasis is mostly by hematogenous spread [18]. Liver is the most common site of secondary tumor metastasis followed by lungs and peritoneum [18]. Due to the paucity of data, there is not enough evidence to establish reliable mortality estimates. However, based on a study by Aggarwal et al. in 2012 only 2 of 11 cases of colon LMS survived in 5-year surveillance [1]. The main cause of death was spreading of the primary tumor and multiple organ failure [1].

Medical therapy is the main course of treatment in mesenchymal GI malignancies, particularly GISTs. However, Due to absence of KITmutations in LMS, tyrosine kinase inhibitors (TKI) are not effective in tumor treatment. Therefore, Surgery is considered as the gold standard treatment for LMS [19]. Since, there are only a few numbers of true LMS cases reported, no standard therapeutic strategy has been established. Radical excision is the most reasonable option since even with low-grade tumors recurrences occurred [20]. According to YTNM Lee et al. all cases of smooth muscle sarcomas, should undergo wide excisional surgery with $4 \mathrm{~cm}$ tumor margin involving mesentery [21].

Although lymph node involvement is very uncommon in LMS, lymph node dissection is recommended due to its highly invasive nature $[3,16]$. Anthracyclines, first-line conventional chemotherapy regimen for soft tissue sarcomas, have minimal to no effect on LMS. A multi-drug regimen of Doxorubicin plus dacarbazine may have some clinical response in treatment. In conclusion, Adjuvant chemotherapy is unnecessary when tumors are completely resected [19]. However, neoadjuvant chemotherapy may decrease the risk of local recurrence in some cases of rectal sarcomas [22]. Furthermore, radiotherapy is completely unbeneficial since LMS is highly radio-resistant [23].

\section{Conclusion}

Herein, we described two rare cases of colon LMS which was confirmed with IHC. The tumors were surgically removed via laparoscopic approach, with wide, tumor-free margins. Due to the rarity of disease, there is not enough information about tumor characteristics. Overall, colonic LMS is a highly invasive neoplasm with poor oncologic outcome.

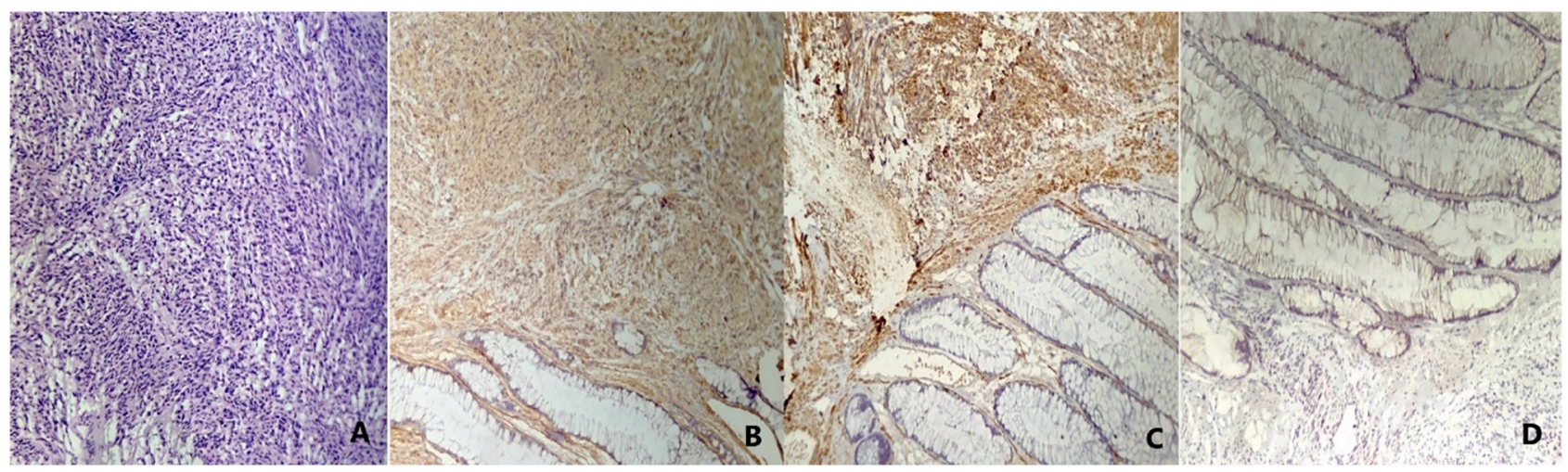

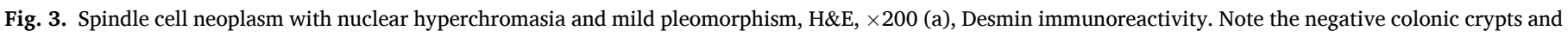
positive tumoral cells for desmin, $\times 400$ (b), Smooth muscle actin, Negative colonic crypts and positive tumoral cells for C-KIT, $\times 400$ (d). 
Table 1

All cases of published colonic LMS after the pre-kit era.

\begin{tabular}{|c|c|c|c|c|c|c|c|c|c|c|c|c|}
\hline Case & Year & Age & Sex & site & $\begin{array}{l}\text { Size } \\
(\mathrm{CM})\end{array}$ & Local recurrence & Metastasis & Survival & c-kit & $\alpha$-SMA & Desmin & CD-34 \\
\hline $1[24]$ & 2000 & 54 & M & $\mathrm{D}$ & 3.2 & $\mathrm{U}$ & $\mathrm{U}$ & Dead & Neg & Pos + & Pos + & Neg \\
\hline $2[24]$ & 2000 & 61 & $\mathrm{M}$ & A & 4.2 & None & None & Alive & Neg & Pos + & $\mathrm{U}$ & $\mathrm{Neg}$ \\
\hline $3[24]$ & 2000 & 75 & M & A & 6.5 & $\mathrm{U}$ & $\mathrm{U}$ & Dead & Neg & Pos + & $\mathrm{U}$ & $\mathrm{Neg}$ \\
\hline $4[24]$ & 2000 & 76 & $\mathrm{~F}$ & $\mathrm{C}$ & 7.8 & $\mathrm{U}$ & $\mathrm{U}$ & Dead & Neg & Pos + & $\mathrm{U}$ & $\mathrm{Neg}$ \\
\hline $5[24]$ & 2000 & 36 & $\mathrm{~F}$ & $\mathrm{~S}$ & 6.5 & None & Lung & Dead & Neg & Pos+ & Pos + & $\mathrm{Neg}$ \\
\hline $6[24]$ & 2000 & 66 & M & A & $\mathrm{U}$ & None & Liver & Dead & Neg & Pos + & $\mathrm{U}$ & $\mathrm{Neg}$ \\
\hline $7[24]$ & 2000 & 41 & M & $\mathrm{C}$ & 7.5 & None & Humerus & Alive & Neg & Pos + & Pos + & $\mathrm{Neg}$ \\
\hline $8[25]$ & 2004 & 65 & M & $\mathrm{D}$ & 10 & None & Positive (U) & Dead & $\mathrm{Neg}$ & Pos + & Pos + & $\mathrm{Neg}$ \\
\hline $9[26]$ & 2004 & 67 & $\mathrm{~F}$ & $\mathrm{~T}$ & 5.7 & None & None & Alive & $\mathrm{Neg}$ & Pos + & $\mathrm{U}$ & $\mathrm{Neg}$ \\
\hline $10[11]$ & 2007 & 77 & $\mathrm{~F}$ & $\mathrm{~S}$ & $\mathrm{U}$ & Positive & None & $\mathrm{U}$ & Neg & Pos + & Pos + & $\mathrm{Neg}$ \\
\hline $11[11]$ & 2007 & 52 & M & $\mathrm{S}$ & $\mathrm{U}$ & None & Liver & $\mathrm{U}$ & Neg & Pos + & Pos+ & $\mathrm{Neg}$ \\
\hline $12[27]$ & 2009 & 74 & $\mathrm{~F}$ & A & 6 & None & Lung & Dead & Neg & Pos+ & $\mathrm{U}$ & $\mathrm{U}$ \\
\hline $13[28]$ & 2011 & 70 & $\mathrm{~F}$ & $\mathrm{~S}$ & 3.7 & None & None & Dead & Neg & Pos+ & Pos + & $\mathrm{Neg}$ \\
\hline $14[28]$ & 2011 & 56 & M & $\mathrm{C}$ & $\mathrm{U}$ & None & Liver & Alive & Neg & Pos + & Pos + & $\mathrm{Neg}$ \\
\hline $15[29]$ & 2012 & 66 & $\mathrm{~F}$ & $\mathrm{~S}$ & 3 & None & Liver & Dead & Neg & Pos+ & Pos+ & $\mathrm{Neg}$ \\
\hline $16[16]$ & 2013 & 94 & $\mathrm{~F}$ & $\mathrm{D}$ & 25 & None & Liver & Dead & Neg & Pos+ & $\mathrm{U}$ & $\mathrm{Neg}$ \\
\hline $17[16]$ & 2013 & 56 & M & $\mathrm{S}$ & 1 & None & $\mathrm{LN}$ & Alive & Neg & Pos+ & $\mathrm{U}$ & $\mathrm{Neg}$ \\
\hline $18[16]$ & 2013 & 78 & $\mathrm{~F}$ & $\mathrm{~s}$ & 8.5 & None & Lung & Dead & Neg & Pos+ & $\mathrm{U}$ & $\mathrm{Neg}$ \\
\hline $19[16]$ & 2013 & 87 & M & $\mathrm{T}$ & 11 & None & None & Dead & Neg & Pos+ & $\mathrm{U}$ & $\mathrm{Neg}$ \\
\hline $20[30]$ & 2013 & 65 & M & $\mathrm{s}$ & $\mathrm{U}$ & None & None & Alive & Neg & Pos+ & $\mathrm{U}$ & $\mathrm{U}$ \\
\hline $21[31]$ & 2014 & 66 & $\mathrm{~F}$ & $\mathrm{~T}$ & 4 & None & None & Alive & Neg & Pos+ & $\mathrm{U}$ & $\mathrm{Neg}$ \\
\hline $22[30]$ & 2014 & 65 & M & $\mathrm{s}$ & $\mathrm{U}$ & None & None & Alive & Neg & Pos+ & $\mathrm{U}$ & $\mathrm{U}$ \\
\hline $23[6]$ & 2015 & 46 & M & $\mathrm{T}$ & 11.8 & Positive & None & Alive & Neg & Pos+ & $\mathrm{U}$ & Neg \\
\hline $24[32]$ & 2015 & 89 & $\mathrm{~F}$ & A & 4.5 & None & Liver & $\mathrm{U}$ & Neg & Pos+ & $\mathrm{U}$ & $\mathrm{U}$ \\
\hline $25[5]$ & 2015 & 54 & M & A & 13 & Positive & None & Alive & Neg & Pos+ & Pos+ & $\mathrm{Neg}$ \\
\hline $26[33]$ & 2015 & 59 & M & A & 10 & None & None & Alive & Neg & Pos+ & Pos+ & Neg \\
\hline $27[34]$ & 2016 & 89 & M & $\mathrm{C}$ & 2.2 & None & None & Alive & Neg & Pos+ & Pos+ & $\mathrm{U}^{\circ}$ \\
\hline $28[35]$ & 2016 & 51 & $\mathrm{~F}$ & $\mathrm{D}$ & 4 & None & None & Alive & Neg & Pos+ & Pos+ & Neg \\
\hline $29[36]$ & 2016 & 44 & M & SF & 8.5 & None & None & Alive & Neg & Pos + & Pos+ & $\mathrm{Neg}$ \\
\hline $30[9]$ & 2017 & 55 & $\mathrm{~F}$ & A & 8 & None & None & Alive & Neg & Pos + & Pos+ & Neg \\
\hline $31[14]$ & 2018 & 57 & $\mathrm{~F}$ & $\mathrm{~S}$ & $\mathrm{U}$ & $\mathrm{U}$ & $\mathrm{U}$ & Alive & Neg & Pos+ & $\mathrm{U}$ & $\mathrm{Neg}$ \\
\hline $32[14]$ & 2018 & 88 & M & A & 6.5 & None & Liver & Dead & Neg & Pos+ & $\mathrm{U}$ & $\mathrm{U}$ \\
\hline $33[37]$ & 2019 & 53 & M & $\mathrm{s}$ & 3.5 & None & None & Alive & Neg & Pos + & Pos + & $\mathrm{Neg}$ \\
\hline $34[3]$ & 2019 & 46 & M & $\mathrm{S}$ & 4.2 & None & None & Alive & Neg & Pos + & Pos + & $\mathrm{Neg}$ \\
\hline 35(case1) & 2019 & 48 & M & $\mathrm{S}$ & 7.2 & None & None & Alive & Neg & Pos+ & Pos+ & Neg \\
\hline 36 (case2) & 2020 & 49 & M & $\mathrm{S}$ & 4 & None & Peritoneum Lungs & Alive & Neg & Pos+ & Pos+ & $\mathrm{Neg}$ \\
\hline
\end{tabular}

Ethics approval and consent to participate

The purpose of this research was completely explained to the patient, and was assured that their information will be kept confidential by the researchers. The present study was approved by the medical ethics committee of the academy.

\section{Funding}

No funding was obtained for this study.

\section{CRediT authorship contribution statement}

$\mathrm{AB}$ - drafted the manuscript and provided images. MS- helped with the draft and reviewed the literature. MM- provided histologic evaluations. RS- Supervisor, provided initial feedback and reviewed the final manuscript (corresponding author). The authors read and approved the final manuscript.

\section{Guarantor}

\section{Ramin Shekouhi M.D}

\section{Consent for publication}

Written informed consent was obtained from the patient for publication of this case report and any accompanying images. A copy of the written consent is available for review by the Editor-in-Chief of this journal.

\section{Availability of data and materials}

All data generated or analyzed during this study are included in this published article. A preprint copy of the article is available in research square (https://doi.org/10.21203/rs.3.rs-214702/v1).

\section{Provenance and peer review}

Not commissioned, externally peer-reviewed.

\section{Declaration of competing interest}

The authors declare that they have no competing interests.

\section{Acknowledgements}

Not applicable.

\section{References}

[1] G. Aggarwal, et al., Primary leiomyosarcomas of the gastrointestinal tract in the post-gastrointestinal stromal tumor era, Ann. Diagn. Pathol. 16 (6) (2012) $532-540$.

[2] S.C. Katz, R.P. DeMatteo, Gastrointestinal stromal tumors and leiomyosarcomas, J. Surg. Oncol. 97 (4) (2008) 350-359.

[3] M. Yahagi, et al., Laparoscopic surgery to treat leiomyosarcomas of the sigmoid colon: a case report and literature review, Surg. Case Rep. 5 (1) (2019) 20.

[4] R.A. Agha, et al., The SCARE 2020 guideline: updating consensus surgical CAse REport (SCARE) guidelines, Int. J. Surg. 84 (2020) 226-230.

[5] P. Kiran, et al., Diagnosis of leiomyosarcoma of colon, J. Cancer Res. Ther. 11 (4) (2015) 1035.

[6] M. Kono, et al., Primary leiomyosarcoma of the colon, Clin. J. Gastroenterol. 8 (4) (2015) 217-222. 
[7] S. Hirota, et al., Gain-of-function mutations of c-kit in human gastrointestinal stromal tumors, Science 279 (5350) (1998) 577-580.

[8] B. Liegl, et al., Monoclonal antibody DOG1. 1 shows higher sensitivity than KIT in the diagnosis of gastrointestinal stromal tumors, including unusual subtypes, Am. J. Surg. Pathol. 33 (3) (2009) 437-446.

[9] J. Yang, Primary leiomyosarcoma in the colon: a case report, Medicine 97 (7) (2018).

[10] M. Miettinen, J. Lasota, Gastrointestinal stromal tumors: review on morphology, molecular pathology, prognosis, and differential diagnosis, Arch. Pathol. Lab. Med. 130 (10) (2006) 1466-1478.

[11] A. Agaimy, P.H. Wünsch, True smooth muscle neoplasms of the gastrointestinal tract: morphological spectrum and classification in a series of 85 cases from a single institute, Langenbeck's Arch. Surg. 392 (1) (2007) 75-81.

[12] G.L. Ma, et al., Epidemiology of gastrointestinal stromal tumors in the era of histology codes: results of a population-based study, Cancer Epidemiol. Prev. Biomarkers 24 (1) (2015) 298-302.

[13] P. Singh, et al., Rectal leiomyosarcoma in association with ulcerative colitis: a rare condition with an unusual presentation, Int. J. Color. Dis. 29 (7) (2014) 887-888.

[14] J.S. Crystal, et al., Primary leiomyosarcoma of the colon: a report of two cases, review of the literature, and association with immunosuppression for IBD and rheumatoid arthritis, Case Rep. Surg. (2018).

[15] R.A. Gladdy, et al., Predictors of survival and recurrence in primary leiomyosarcoma, Ann. Surg. Oncol. 20 (6) (2013) 1851-1857.

[16] H. Yamamoto, et al., Clinicopathological features of primary leiomyosarcoma of the gastrointestinal tract following recognition of gastrointestinal stromal tumours, Histopathology 63 (2) (2013) 194-207.

[17] H. Yamamoto, et al., Infantile intestinal leiomyosarcoma is prognostically favorable despite histologic aggressiveness: case report and literature review, J. Pediatr. Surg. 39 (8) (2004) 1257-1260.

[18] W. Faraj, et al., Liver resection for metastatic colorectal leiomyosarcoma: a single center experience, J. Gastrointest. Oncol. 6 (5) (2015), E70.

[19] Group, E.E.S.N.W, Soft tissue and visceral sarcomas: ESMO Clinical Practice Guidelines for diagnosis, treatment and follow-up, Ann. Oncol. 25 (suppl_3) (2014) p. iii102-iii112.

[20] E.N. Ng, R.E. Pollock, M.F. Munsell, E.N. Atkinson, M.M. Romsdahl, Prognostic factors influencing survival in gastrointestinal leiomyosarcomas: implications for surgical management and staging, Ann. Surg. 215 (1992) 68-77.
[21] Y.-T.N.M. Lee, Leiomyosarcoma of the gastro-intestinal tract: general pattern of metastasis and recurrence, Cancer Treat. Rev. 10 (2) (1983) 91-101.

[22] P. Luna-Pérez, et al., Colorectal sarcoma: analysis of failure patterns, J. Surg. Oncol. 69 (1) (1998) 36-40.

[23] M. Koczkowska, et al., Primary leiomyosarcoma of the mesentery in two sisters: clinical and molecular characteristics, Pol. J. Pathol. 64 (1) (2013) 59-63.

[24] M. Miettinen, et al., Gastrointestinal stromal tumors and leiomyosarcomas in the colon: a clinicopathologic, immunohistochemical, and molecular genetic study of 44 cases, Am. J. Surg. Pathol. 24 (10) (2000) 1339-1352.

[25] L. Insabato, et al., Malignant gastrointestinal leiomyosarcoma and gastrointestinal stromal tumor with prominent osteoclast-like giant cells, Arch. Pathol. Lab. Med. 128 (4) (2004) 440-443.

[26] A. Michalopoulos, et al., Colorectal gastrointestinal mesenchymal tumours. report of a stromal case of the rectum (GIST) and a leiomyosarcoma of the transverse colon, Techn. Coloproctology 8 (1) (2004) s155-s157.

[27] C.M. Alvite, et al., Leiomyosarcoma of the colon with lung metastases as the first manifestation, Rev. Esp. Enferm. Dig. 101 (2) (2009) 145.

[28] T. Resch, et al., Leiomyosarcoma of the colon: unresolved issues of a rare but highly aggressive malignancy, Am. Surg. 77 (4) (2011) E62.

[29] Y. Hamai, et al., Leiomyosarcoma of the sigmoid colon with multiple liver metastases and gastric cancer: a case report, BMC Gastroenterol. 12 (1) (2012) 98.

[30] A.A. Samie, et al., Leiomyosarcoma of the sigmoid colon: a rare cause of intestinal intussusception, J. Gastrointest. Cancer 45 (1) (2014) 6-9.

[31] A. Yaren, et al., Primary mesenchymal tumors of the colon: a report of three cases, Turk. J. Gastroenterol. 25 (3) (2014) 314-318.

[32] L. Granero-Peiró, et al., Leiomyosarcoma of the ascending colon: a rare tumor with poor prognosis, Rev. Esp. Enferm. Dig. 107 (9) (2015) 580-581.

[33] V. Janevski, et al., Leiomyosarcoma of the colon, Med. Pregl. 68 (11-12) (2015) 413-417.

[34] V.M. Kim, L. Goicochea, S.H. Fang, Case report: collision tumour of colon leiomyosarcoma and adenocarcinoma, J. Clin. Diagn. Res. 10 (6) (2016) p. PD03.

[35] D. Akutsu, et al., A rare case of colonic leiomyosarcoma in association with ulcerative colitis, Intern. Med. 55 (19) (2016) 2799-2803.

[36] B. Jideh, T. Yang, I.B. Turner, Rectal bleeding due to leiomyosarcoma, Clin. Gastroenterol. Hepatol. (2017) e1-e2.

[37] S. Devriendt, G. Leman, F. Vanrykel, Primary leiomyosarcoma of the colon: a case report and review of the literature, Acta Chir. Belg. (2019) 1-4. 